Appl. Phys. 8, 163-171 (1975)

(C) by Springer-Verlag 1975

\title{
Phonon Emission Spectra of Thin Metallic Films
}

\author{
Wolfgang Frick ${ }^{\star}$, Dieter Waldmann, and Wolfgang Eisenmenger \\ Physikalisches Institut, Teilinstitut 1, Universität, D-7 Stuttgart, Fed. Rep. Germany
}

Received 28 April 1975 / Accepted 5 June 1975

\begin{abstract}
Thin metallic films evaporated on an $\mathrm{Al}_{2} \mathrm{O}_{3}$-single crystal and cooled to liquid helium temperatures are heated by short electric current pulses. The high frequency part of the emitted phonons is detected by calibrated superconductive tunneling junctions on the opposite surface of the substrate. The observed phonon detector signal amplitude is compared with theoretical models taking account of the boundary conditions for elastic waves in the film. It is found that the phonon spectrum emitted perpendicularly to the substrate-film boundary depends strongly on the thickness of the heater film.
\end{abstract}

Index Headings: Thin films - Phonon scattering - Heat pulse experiments

Phonon propagation experiments in solids meanwhile cover the entire frequency range up to the highest lattice frequencies. Ultrasonic waves, i.e. monochromatic and coherent phonons limited to frequencies below $10^{10} \mathrm{~Hz}$ are usually generated and detected by piezoelectric transducers [1]. Infrared absorption or Raman scattering techniques are applied in the range of optical phonons. Quasimonochromatic acoustical phonons between $10^{11}$ and $10^{13} \mathrm{~Hz}$ can be generated and detected by means of superconductive tunneling junctions [2]. Experiments with thermal phonon radiators were first described by Gutfeld and Nether$\cot [3]$. The phonons emitted from a thin film metallic heater into the crystal-substrate cover a wide frequency band analogous to the Planck spectrum of photons in black body radiation. As in Wien's law the frequency position of the spectral maximum depends on temperature according to $h v_{m} \approx 2.82 \mathrm{kT}$ for temperatures below the Debye temperature and the total radiation power follows the $T^{4}$ law of Stephan-Boltzmann. Weis [4] calculated the total phonon radiation from the heater in analogy to Debye's modified theory in the limit of heater dimensions large compared with the phonon wavelength. He was able to verify experimen-

* Present address: Institut für Halbleitertechnik, D-7 Stuttgart 1, Fed. Rep. Germany. tally the $T^{4}$ law also with respect to the calculated prefactor. The good agreement between theory and measurement justifies the acoustic mismatch model [5] for phonon transmission.

In this paper we report on heat pulse measurements using extremely "thin heater" films with thickness values down to $60 \AA$ evaporated on sapphire crystals [6]. Since the film thicknesses in these experiments are of the order of magnitude of the phonon wavelength or smaller the phonon density of states is changed by finite thickness effects depending on the acoustic boundary conditions at the free surface of the "thin heater" and at the interface to the substrate. Several "thin heater" models with different boundary conditions are discussed. One of these models describes our "thin heater" measurements satisfactorily well whereas significant deviations between experiment and the "thick heater" calculations [4] are found. In order to obtain information on the phonon spectrum emitted by the constantan heater superconductive tunneling junctions [2] of Sn and $\mathrm{Al}$ (Al: oxygen enhanced energy gap) have been used as detector. These junctions have high-pass properties being sensitive only for phonons with energies exceeding $2 A$ of the superconductor. From the detector signal dependence on heater power and crystal temperature we obtain information on 
characteristic properties of the energy distribution in thin films, on the mean free phonon path in constantan and on the existence of characteristic boundary conditions.

\section{Experiment}

A constantan film is evaporated on one mechanically polished end surface of a cylindrical $c$-cut sapphire single crystal of $2 \mathrm{~cm}$ diameter and $0.7 \mathrm{~cm}$ thickness (Fig. 1). Two indium films serve as contacts and define the heater area. For different thickness values of the film length and width of the heater are chosen such as to result in $50 \mathrm{ohms} \mathrm{sample} \mathrm{resistance.} \mathrm{The} \mathrm{heater} \mathrm{area}$ varies between 0.25 and $2.2 \mathrm{~mm}^{2}$. The superconductive tunneling detector with $1 \mathrm{~mm}^{2}$ area on the opposite face of the crystal consists of two films of $2000 \AA$ thickness separated by an oxide barrier of about $10 \AA$. Heater and tunneling junction are evaporated under high vacuum conditions (pressure $<10^{-5}$ Torr). The film thickness has been determined by a quartz oscillator thickness monitor and by Tolansky multiple beam interference. Electron microscopy indicated that films exceeding $80 \AA$ thickness are homogeneous whereas films below $60 \AA$ develop an island like structure of their surface which was supported by the negative temperature gradient and the extremely strong thickness dependence of the resistivity of these very thin films. In addition, electron microscopic inspection revealed a crystal surface roughness small compared to our experimental phonon wavelength range.

The measurements have been performed in the temperature range from 1 to $2 \mathrm{~K}$ in vacuum or in contact with liquid helium. All data have been taken under pulsed conditions. The signal current of the junction, biased below $24 / e$, is amplified and sampled via

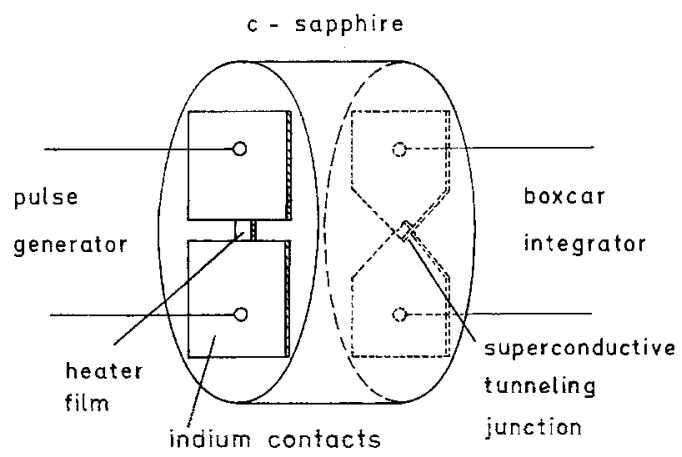

Fig. 1. Diagram of the sample
Boxcar integrator. The signal can be $x-y$ recorded as a function of time (lifetime and time of flight measurements) or as a function of heater power.

\section{Phonon Emission Spectra}

At the present time, the precise mechanism of phonon generation is not fully understood. A microscopic description how the phonon distribution develops is therefore not available. So far, Kappus and Weis [7] have estimated, that in a $100 \AA$ metal film a stationary phonon distribution is established some ns after current onset. This thermal distribution does not change during the whole pulse of some $\mu \mathrm{s}$ and the rate of phonons generated is equal to the rate of emitted phonons. If

$e^{(10)}<\frac{4 d}{A_{\text {int }}}$

holds [8], where $e^{(10)}$ is the emissivity of phonons from the heater " 1 " (thickness $d$ ) to the substrate " 0 " and $A_{\text {int }}$ is the mean free path of phonons in the heater, a thermal equilibrium distribution can be defined with the heater temperature $T_{1}$. The theoretical values of several $1000 \AA$ for the mean free path in constantan [8] appear as too large. The emissivities $e^{(10)}$ for the interface constantan/sapphire are calculated by Weis [4] to 0.216 for longitudinal and 0.176 for transversal elastic waves. These values allow a thermal equilibrium distribution only for films thicker than $189 \AA$. In contrast to this our experimental results indicate that also in thinner films the concept of an equilibrium distribution corresponding to a certain heater temperature $T_{1}$ is allowed.

To calculate the phonon density of states we regard the heater as a free body infinite in two dimensions $(x, y)$, but with finite thickness $d$ in the third dimension (z). While the density of $q$-states in planes parallel to $q_{z}=0$ can be taken as continuous [4], we only consider the $z$-dependence of all components of stress and strain. According to the boundary conditions only discrete wavelength values are allowed, i.e. discrete values of the $z$-components of the wave-vectors. Different basically possible boundary conditions are listed in Table 1.

Model 1 corresponds to cyclic boundary conditions as valid for heaters that are infinite in all dimensions with the exception that all states with $q_{z}=0$ are missing. This seems to be somewhat arbitrary at this moment, but will be discussed later. 
Table 1. Models of standing waves in the z-direction at different boundary conditions

\begin{tabular}{|c|c|c|c|c|}
\hline Model & Boundary condition & Elongation & Allowed wave length & Allowed wave vectors \\
\hline Model 1 & periodic & & $n \cdot \hat{\lambda}=d$ & $q_{z}=n \cdot \frac{2 \pi}{d} ; \quad n=1,2,3, \ldots N$ \\
\hline Model 2 & weakly coupled boundaries & & $n \cdot \frac{\lambda}{2}=d$ & $q_{z}=n \cdot \frac{\pi}{d} ; \quad n=1,2,3, \ldots N$ \\
\hline Model 3 & clamped boundaries & & $n \cdot \frac{\lambda}{2}=d$ & $q_{z}=n \cdot \frac{\pi}{d} ; \quad n=0,1,2, \ldots N-1$ \\
\hline Model 4 & $\begin{array}{l}\text { free boundary at one side and } \\
\text { clamped boundary at the other side }\end{array}$ & & $n \cdot \frac{\lambda}{2}+\frac{\lambda}{4}=d$ & $q_{z}=\left(n+\frac{1}{2}\right) \cdot \frac{\pi}{d} ; \quad n=0,1,2 \ldots N-1$ \\
\hline
\end{tabular}

In Model 2 we make the assumptions for a free or weakly coupled boundary on both sides of the heater like the unloaded ends of a free oscillating bar. The stress at the surfaces $z=0$ and $z=d$ vanishes and therefore no lattice vibrational states with $q_{z}=0$ can be excited.

Model 3 is valid for strong coupling or "clamped" boundary conditions on each side of the heater, according to media with comparatively high mechanical wave impedance being in contact with the heater (substrate, helium layer, etc.). Here the stress is constant at the surfaces corresponding to a minimum of the displacement. The only exception is the static case with constant stress and displacement across the heater thickness. Therefore, phonon states with $q_{z}=0$ now are permitted in analogy to the fundamental mode of Kund's tube. The phonon distribution basing on this model contains a considerable part of long wavelength phonons, propagating parallel to the interface and having the possibility to escape into the crystal.

Contrary to this model, Model 1 and 2 exclude the excitation and radiation of such low frequency phonons parallel to the interface. These models contain implicitly the existence of a limiting angle of total reflection.

Model 4 corresponds to acoustic strong coupling or clamped boundary conditions on one side and free boundary conditions on the other side of the layer, i.e. $q_{z}=0$ is forbidden, too. Therefore, no plane waves propagating parallel to the surface can be excited or radiated. Since the calculations are analogous for all models, the discussion will be limited to Model 2 as an example. The location of the phonon states in $q$-space is demonstrated in Fig. 2 .

While the states in $x$ and $y$ direction are packed very closely corresponding to the Debye mode, the distances of the planes $q_{z}$ in the $z$-direction are long (see Table 1). Counting the states in a spherical shell of radius $q$ and thickness $d q$, we get the phonon density of states in

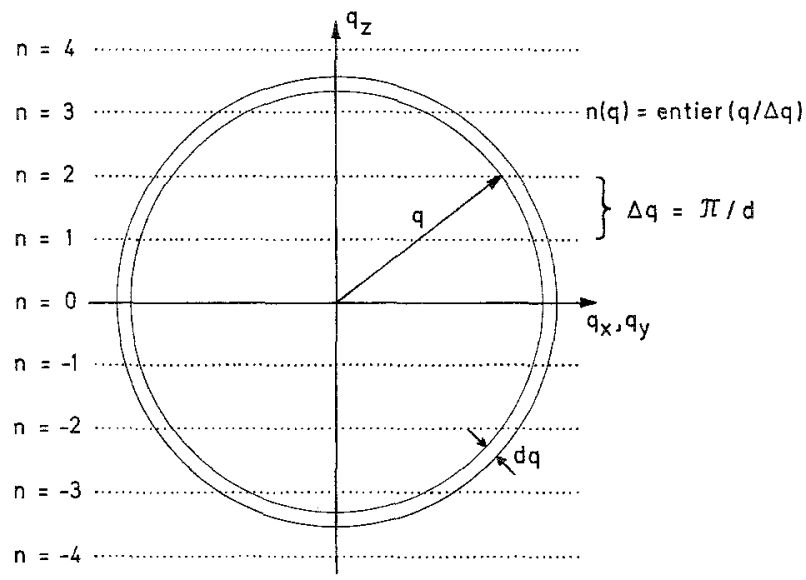

Fig. 2. Phonon states in $q$-space according to Model 2 
$q$-space in a volume $V$

$D^{(2)}(q)=\frac{V q}{2 \pi d} \operatorname{entier}(q d / \pi)$.

If we consider only frequencies below $1 \mathrm{THz}$, we can assume a linear dispersion law [4]

$v=\frac{q c_{\sigma}^{(1)}}{2 \pi}$

where $c_{\sigma}^{(1)}$ is the phase velocity for phonons of polarization $\sigma$ in the heater " 1 ". At temperature $T_{1}$ there are excited

$m_{\sigma}^{(1)}\left(v, d, T_{1}\right)=\frac{2 \pi \nu}{c_{\sigma}^{(1) 2} d} \operatorname{entier}\left(2 v d / c_{\sigma}^{(1)}\right) f\left(v, T_{1}\right)$

phonons per unit volume and frequency interval $(v, v+d v)$. The Bose-Einstein distribution function

$f(v, T)=[\exp (h v / k T)-1]^{-1}$

is valid for phonons under the conditions mentioned above. With the assumption of an isotropic angle distribution for the excited phonons, we get a net phonon radiation [16] according to $[4,5]$ from the heater " 1 " with area $F$ into the crystal " 0 ".

$$
\begin{aligned}
n_{\sigma}^{(10)}\left(v, d, T_{1}, T_{0}\right)= & \frac{\pi F e_{\sigma}^{(10)}}{2 c_{\sigma}^{(1)} d} v \operatorname{entier}\left(2 v d / c_{\sigma}^{(1)}\right) \\
& \cdot\left[f\left(v, T_{1}\right)-f\left(v, T_{0}\right)\right],
\end{aligned}
$$

where the function entier $(x)$ means the greatest natural number not exceeding $x$. This result, derived explicitely for the case of Model 2, is valid in similar form for all other models. With (6) we obtain the spectral energy flux of the radiated phonons

$p_{\sigma}^{(10)}\left(v, d, T_{1}, T_{0}\right)=h v n_{\sigma}^{(10)}\left(v, d, T_{1}, T_{0}\right)$.

This function is plotted in Fig. 3a for infinite thickness of the heater and for two different heater temperatures (modified Debye model, model of Weis [4]). The frequency distribution corresponds to the Planck spectrum for photons. The spectrum calculated in Model 2 is shown, too, here for a fixed thickness $d=140 \AA$. Low frequency phonon states are forbidden (except in Model 3). At discrete frequencies we have sharp steps in the number of excited phonon states. In Fig. $3 b$ the emitted phonon power density is shown for various thickness values $d$. Note the relative increase of high frequency phonon contributions with decreasing thickness $d$.

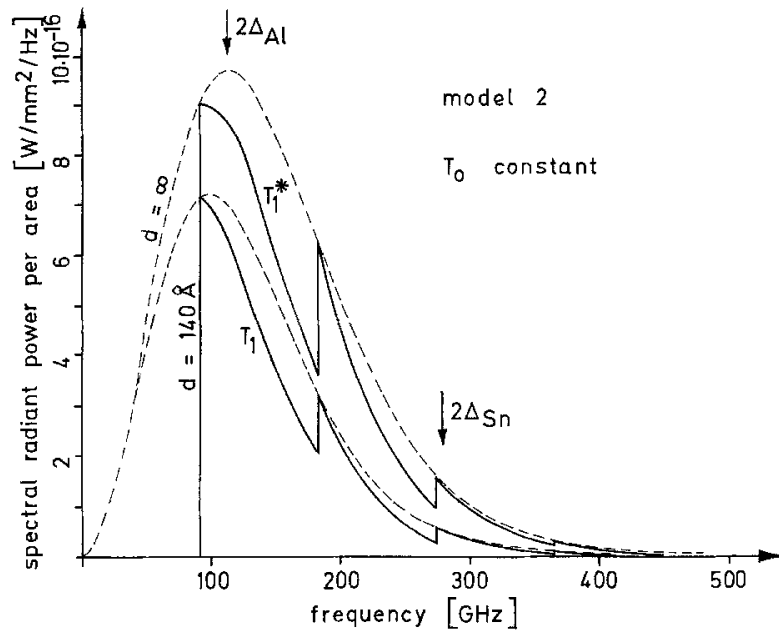

Fig. 3a. Spectral radiant phonon power for two heater temperatures $T_{1}, T_{1}^{*}>T_{1}$ calculated after the thick limit model-and after Model 2 - For a finite thickness $(d=140 \AA)$ we have an increased heater temperature $T_{1}^{*}$ to emit the same total phonon power as in the thick limit $(d \rightarrow \infty)$. Note the different location of the energy gap of the two tunneling junctions

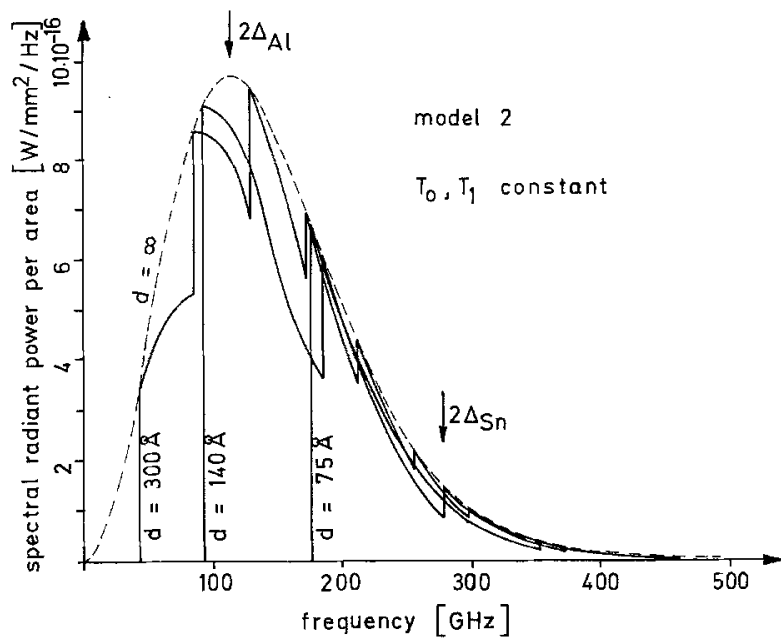

Fig. 3b. Spectral radiant phonon power after Model 2 for different thicknesses $d$ of the heater films. Heater temperature $T_{1}$ and crystal temperature $T_{0}$ are held constant

From (7) the total power of emitted phonons can be written

$P_{\text {tot }}\left(d, T_{1}, T_{0}\right)=\sum_{\sigma} \int_{0}^{v_{\sigma \max }} p_{\sigma}^{(10)}\left(v, d, T_{1}, T_{0}\right) d v$

The integration has to be extended to the cutoff frequency, which can be determined from the number $N$ of atoms per volume $V$ and the phase velocity 
$c_{\sigma}^{(1)}$ [9]. $P_{\text {tot }}$ of (8) is equal to the electric power $P_{\text {el }}$ supplied to the heater.

Regarding Fig. 3a we see that for thin films and decreasing thickness $d$ the heater temperature $T_{1}$ necessarily increases in order to result in the same total phonon energy flux as in the case of infinite thickness. According to (7) this results in an increased rate of high frequency phonon emission (again with the exception of Model 3). As the detector is only sensitive to phonons with frequencies above $v_{24}=2 \Delta / \mathrm{h}$ low frequencies do not contribute to the signal. Thus the detectable phonon rate escaping from the generator is

$n_{2 \Delta}^{\mathrm{gen}}\left(d, T_{1}, T_{0}\right)=\sum_{\sigma} \int_{v_{2} \Delta}^{v_{\sigma \max }} n_{\sigma}^{(10)}\left(v, d, T_{1}, T_{0}\right) d v$.

Since $n_{\sigma}^{(10)}(v)$ decays exponentially at higher frequency only phonons just above $v_{24}$ must be regarded. Eliminating $T_{1}$ in (8) and (9) we get the generation rate of $2 \Delta$-phonons $n_{2 \Delta}^{\mathrm{gen}}\left(P_{\mathrm{e}}, d, T_{0}\right)$ as a function of the electric power $P_{\mathrm{e} 1}$, crystal temperature $T_{0}$ and heater thickness $d$. Numerical computations for all models are plotted as function of heater power.

Figure 4 shows the result for the case of Model 2 with different values of the heater thickness including the

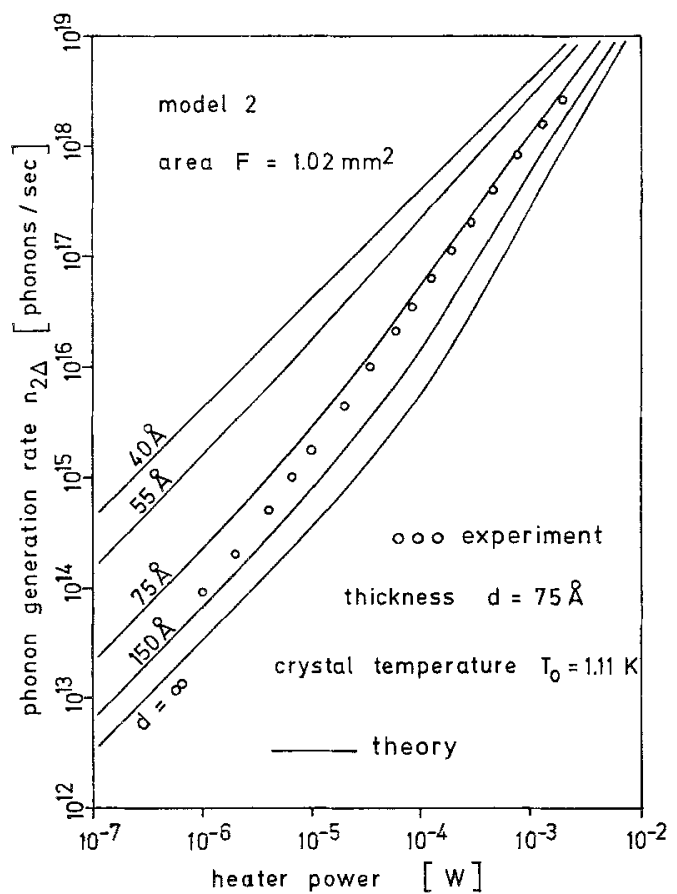

Fig. 4. Phonon generation rate $n_{24}^{\mathrm{gen}}$ versus total heater power $P_{\mathrm{el}}$ calculated for different thicknesses $d$ after Model 2, compared with measurements using a sample of $75 \AA$ thickness. Experimental points are corrected with the factor $1 / \alpha_{v}=10$ limit $d \rightarrow \infty$. In this "thick limit" the dependence of the generation rate of phonons with energy equal to or above $2 \Delta$ ( $\mathrm{Sn}$-detector) on heater power is qualitatively described as follows: At power levels below $10^{-5} \mathrm{~W}$ the heater temperature $T_{1}$ remains close to the crystal temperature $T_{0}$. The shape of the emitted phonon spectrum does not change and the spectral intensity in the entire frequency range including the 24-band increases in proportion to the heater power. In the power range from $10^{-5}$ to $10^{-1} \mathrm{~W}$ the heater temperature exceeds the ctystal temperature and the frequency of the spectral maximum at $v_{m}\left(T_{1}\right)$ shifts from below $2 \Delta / h$ to frequencies above $2 \Delta / h$, thus leading to a stronger signal dependence on heater power than before. At power levels above $10^{-1} \mathrm{~W}$ $v_{m}\left(T_{1}\right)$ is sufficiently large compared to $2 \Delta / h$ and the detectable phonon emission rate again varies in proportion to $P_{\text {tot }}$. Note: The constant of proportiona-

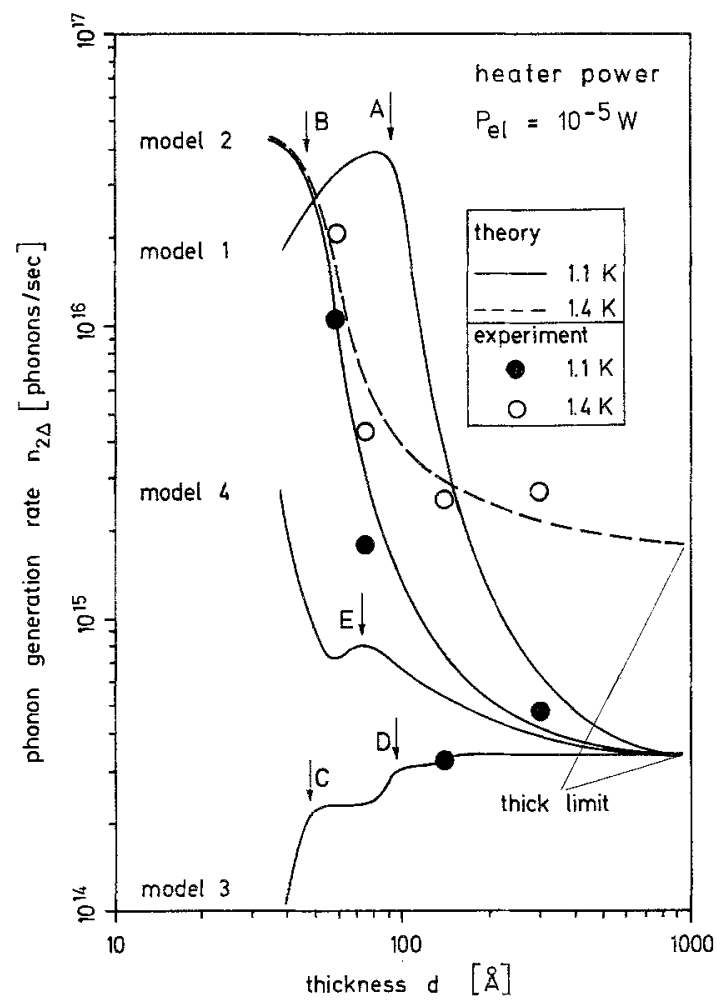

Fig. 5. Phonon generation rate $n_{2 A}^{\text {gen }}$ versus heater thickness $d$ at constant heater power for two crystal temperatures calculated for all models. Note, the experiment is in agreement with the calculations after Model 2. If a step in the transverse phonon frequency spectrum (see Fig. 3b) according to a certain heater thickness $d$ is equal to the energy gap $2 \Delta$ of the detector there appears an additional structure in the curves: $A-1$ st step of Model 1, B-1st step of Model 2, $C-1$ st step of Model 3, D-2nd step of Model 3, E-2nd step of Model 4 
lity is by orders of magnitude larger than before, since most phonon energies are now contributing to the signal.

For "thin heater" films the lack of low energy states (with the exception of Model 3) results in a shift of the radiated spectrum to higher frequencies and a corresponding enhancement of the effective temperature $T_{1}$ and $2 \Delta$ phonon radiation. For sufficiently high heater power or thickness all models converge into the thick film limit. This is more clearly demonstrated by plotting the emitted phonon rate $n_{2 A}^{\text {gen }}$ versus $d$ at constant total power $\left(P_{\text {tot }}=10^{-5} \mathrm{~W}\right)$ for all models (Fig. 5). The thickness dependence in Models 1 to 4 contains additional structure as consequence of the first (or second) step of the transverse phonon spectral distribution reaching the energy gap $2 \Delta$ at certain thickness values (see Fig. 5). The influence of $d$ in Model 3 is weaker and in the opposite direction corresponding to the strong contribution of low frequency phonons. As Fig. 4 and 5 indicate, the phonon generation rate at low total power in Model 2 and thin films $(40 \AA)$ is two orders of magnitude higher than in the thick film limit. Figure 6 shows the generation rate versus total power for different crystal temperatures $T_{0}$. In addition, the crystal temperature

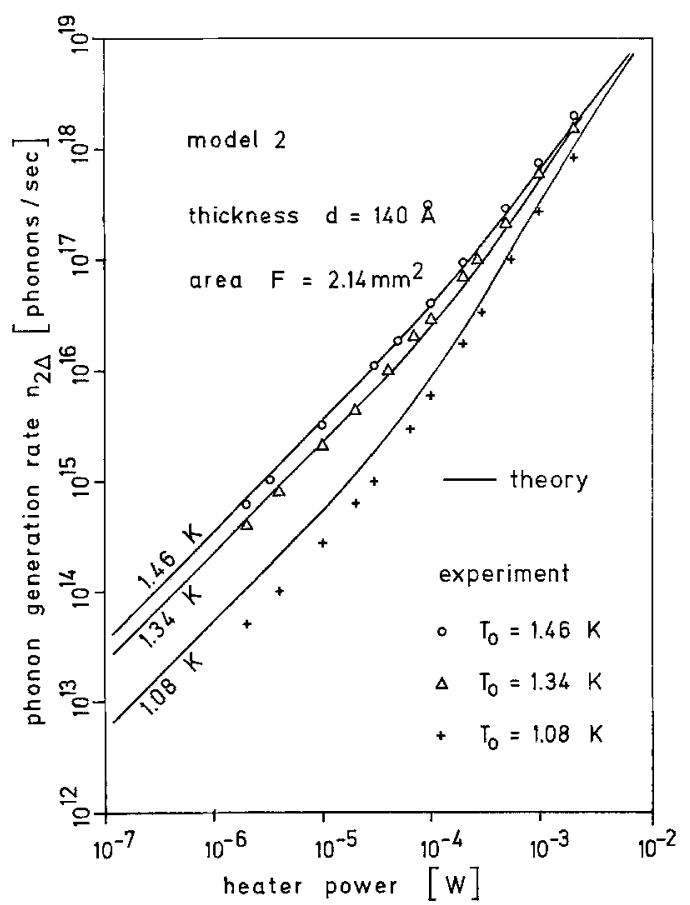

Fig. 6. Phonon generation rate $n_{2 A}^{\text {gen }}$ versus total heater power $P_{\text {el }}$ calculated for a thickness $d=140 \AA$ for different crystal temperatures $T_{0}$ after Model 2 and compared with the experiment

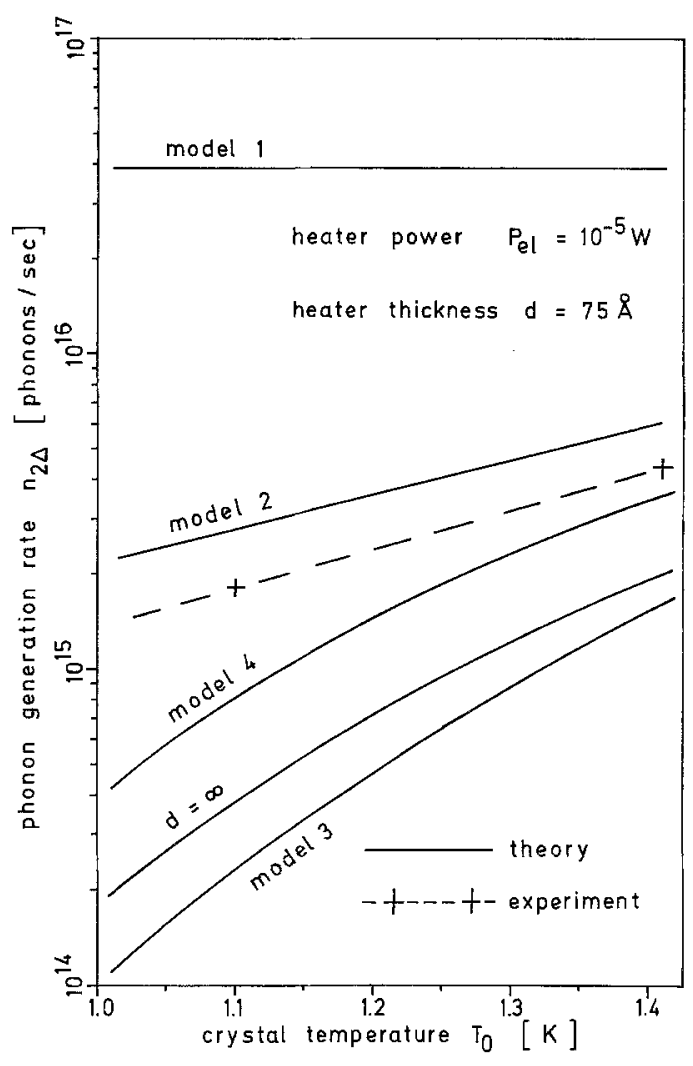

Fig. 7. Calculated phonon generation rate $n_{2 A}^{\text {gen }}$ of all models versus crystal temperature $T_{0}$

dependence of the phonon rate for Models 1 to 4 has been calculated for the thickness $d=75 \AA$ and a constant total power $10^{-5} \mathrm{~W}$ in Fig. 7. Model 1 shows independence from crystal temperature $T_{0}$ for thickness values below $100 \AA$. All other models including the thick limit case result in a monotonic increase of $2 \Delta$ phonon radiation with crystal temperature $T_{0}$.

In order to find the most appropriate model, measurements of the dependence of $n_{24}^{\text {gen }}$ on heater thickness $d$ and crystal temperature $T_{0}$ are necessary.

In addition to these model calculations, we estimated the influence of corresponding phonon surface states [10]. Even under the assumption of perfect matching conditions to the free crystal surface along the edge of the heater area there is no significant contribution of surface phonons for heaters thicker than $50 \AA$.

\section{Phonon Detection and Measurements}

The thermal tunneling current $J_{\text {th }}$ in a superconductive tunneling junction with area $A$ biased at $\Delta / e<V<2 \Delta / e$ depends in good approximation linearly on the 
quasiparticle density $\varrho_{\text {th }}$

$J_{\mathrm{th}}(T)=A C \underline{\mathrm{th}}_{\mathrm{h}}(T)$

with

$\varrho_{\mathrm{th}}(T)=2 N_{0}(2 \pi \Delta k T)^{1 / 2} \exp (-\Delta / k T)$,

where $N_{0}$ is the electron density of states at the Fermi level in normal metals. The constant of proportionality $C$ can be determined by thermal calibration, e.g. measurement of the tunneling current $J_{\text {th }}$ as function of temperature $T$. Since the mean free path of $2 A$ phonons against pair breaking is smaller than the detector film thickness $d_{t u}=4000 \AA$ all $2 \Delta$ phonons incident in the detector contribute to the quasiparticle density increase $\Delta \varrho$ of [11]

$\Delta \varrho=\frac{2 n_{2 \Delta}^{\mathrm{det}} \tau_{\exp }(T)}{V_{t u}}$

Here $\tau_{\text {exp }}(T)$ is the effective quasiparticle recombination lifetime including reabsorption of recombination phonons and $V_{t u}=A \cdot d$ is the junction volume. According to (10) the phonon injected quasiparticles cause a signal current (dynamical short-circuit current).

With the assumption of a $r^{-2}$ dependence of the phonon intensity the number of phonons $\geqq 2 A$ generated can be determined experimentally from the signal current $J_{S}$

$n_{2 \Delta}^{\mathrm{gen}}=\frac{\pi r^{2} d_{t u}}{2 \Delta \tau_{\exp }(T) C} J_{S}$

After determining the tunneling constant $C$ and the effective lifetimes of the quasiparticles $\tau_{\exp }(T)\left(\tau_{\exp } \approx\right.$ $0.2 \ldots 3 \mu \mathrm{s})$ from pulse signal decay measurements [11] we record the signal current $J_{S}$ as a function of the heater voltage which gives us the experimental curve $n_{24}^{\mathrm{gen}}\left(P_{\mathrm{el}}\right)$. By variation of the crystal temperature $T_{0}$ and film thickness $d$ we compare experiment with calculations. Measurements of Trumpp et al. [12] using the method of Eisenmenger and Dayem [2] with $\mathrm{Si}$ and $\mathrm{Al}_{2} \mathrm{O}_{3}$ substrates show a discrepancy between experiment and theory indicating phonon losses during transmission. They found a phonon yield ratio of

$\alpha_{V}=n_{2 \Delta}^{\text {exp }} / n_{2 . \Delta}^{\text {theor }}=0.1$

confirmed also in other experiments. To compare with our model calculation all measured signal amplitudes are, therefore, corrected with the factor
$1 / \alpha_{V}=10$. For high pulse power or thick films, i.e. vanishing influence of the thickness, the experimental results agree with the "thick limit" of all models (see Figs. 4 and 5).

\section{Experimental Results and Discussion}

The experimental dependence of the generator phonon rate on heater power is introduced in Fig. 4 here for a heater with $d=75 \AA$ corrected by the factor of 10 as mentioned above. Qualitative agreement between the theoretical curve (Model 2) and the experimental results, especially for heater thicknesses below $100 \AA$, in contrast to the calculated values for the Models 1, 3,4 is found. This follows more clearly from Fig. 8 where the measurement on the $75 \AA$ heater is compared with all model calculations at the same thickness value for the phonon generation rate as function of the heater power. Only at lower heater powers the agreement with Model 2 decreases in favour of Model 4.

The most critical check of the different models is provided by introducing the results of measurements for different $d$ values in Fig. 5 showing the calculated

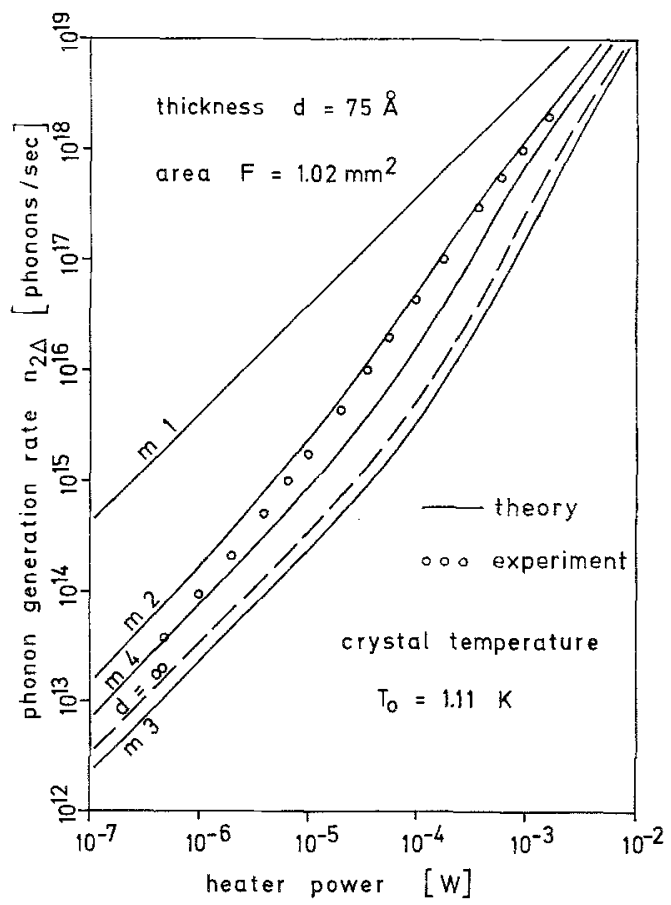

Fig. 8. Phonon generation rate $n_{24}^{\text {gen }}$ versus total heater power calculated at crystal temperature $T_{0}=1.11 \mathrm{~K}$ and thickness $d=75 \AA$ for all models (-) including thick limit (----). Note the agreement with Model 2 
phonon rate as function of film thickness at a fixed power of $10^{-5} \mathrm{~W}$. Again the experimental results for the two temperature ranges 1.1 and $1.4 \mathrm{~K}$ are described best by Model 2 . The agreement between measurement and calculation in the thick limit (Weis [4]) confirms our method for obtaining absolute phonon rates. We note that the experimental results are obtained in vacuum. With liquid helium contact to the sample instead, the phonon emission rate into the crystal is reduced by one order of magnitude confirming the anomalous high phonon transmission into liquid helium according to the Kapitza anomaly (compare also [12]).

Further comparisons of experimental results with calculation are found in Figs. 6 and 7. Figure 6 shows the phonon rate as function of heater power for Model 2 and $d=140 \AA$ with crystal temperature $T_{0}$ as parameter. The experimental values for a heater of the same thickness are in satisfactory agreement with theory. Figure 7 shows the experimental phonon rate and the phonon rates for the different models including the limit $d \rightarrow \infty$ as a function of the crystal temperature $T_{0}$. For the heater power level of $10^{-5} \mathrm{~W}$ and a film thickness $d=75 \AA$ again the experiment is close to Model 2 especially with respect to the slope of the temperature dependence.

Summarizing our experimental results we find: at low power levels phonon radiation from thin heater films deviates significantly from the results predicted for infinite $d[4]$. The dependencies of phonon generation rate on heater power $P_{\mathrm{e}}$; on thickness $d$, and on the crystal temperature $T_{0}$ are satisfactorily described by Model 2, whereas the Models 1, 3, 4 show more or less strong deviations from experiment. Note, however, that in all models simplified boundary condition are used.

Although at low heater powers nearly all emitted phonon energies are below $2 \Delta_{S n}$ (see Fig. 3a, b) the low frequency phonons do not contribute to the signal as assumed throughout our calculations. This agrees with theoretical results of Bobetic [13] and Pippard [14] and with the extrapolation of ultrasonic absorption data [15] both leading to an absorption $<0.02$ for low frequency phonons in our $S n$ detector films.

In a further experiment with a tunneling junction of $\mathrm{Al}$ (oxygen background evaporation) and a heater of $85 \AA$ thickness nearly all phonons radiated have energies above $2 \Delta_{\mathrm{Al}}$. As the number of absorbed phonons in the Al-detector at low heater powers is much higher than in Sn-junctions (see Fig. 3a, b), we observed a correspondingly stronger increase in detector temperature or signal amplitude. The experimental results obtained with the Al-detector again show satisfactory agreement with Model 2.

\section{Conclusion}

The different models especially Model 2 as being supported by our experiments are now discussed with respect to their physical significance. In all models the excited frequency spectrum is strongly anisotropic.

Phonons which are radiated perpendicularly to the heater area have a low energy cut off frequency with the exception of Model 3. For Model 2 this cut off frequency amounts to

$v_{c}=\frac{c_{\sigma}^{(1)}}{2 d}$.

Low frequency phonons with propagation direction parallel to the heater surface may be excited but there is no positive or negative experimental evidence for this, since no radiation under large angles of incidence in the heater can be observed.

Our experimental results clearly indicate that periodic boundary conditions as used in Model 1 are not suitable to describe an influence of thickness. However, one expects that rigid coupling on the crystal side and soft coupling on the side to the vacuum (Model 4) would approximate the real conditions. The experiments instead are in favour of Model 2, i.e. soft coupling on both ends. At the interface heater/substrate this can be explained by total reflection according to a step in phase of the order of $\pi$, phonon transmission being limited to a small cone within the critical angle of total reflection. Our results indicate that the theory of the elastic continua including limited angle of total reflection can be applied also to very thin metal layers. We note that electron microscope inspection indicated that the roughness of the crystal surfaces of our samples is small compared to the wavelength of the phonons.

Furthermore, the results of experiment allow a rough estimation of the mean free phonon path in constantan at temperatures of about $1 \mathrm{~K}$. Since we have not observed any deviation from thermal equilibrium between electrons and phonons for samples with $d \geqq 75 \AA$ our assumption of an unique occupation temperature appears justified. Thus, from (1) (compare [8]) $A_{\text {int }}$ with $1500 \AA$ results as an upper limit for the phonon mean free path in constantan. 
We finally suggest the possible use of extremely thin heater films for incoherent phonon generation in smaller high frequency bands than attainable with heaters in the "thick limit".

Acknowledgement. The authors are grateful to Prof. O. Weis for stimulating discussions. Thanks also to Dr. K. Laßmann and H. J. Trumpp for their helpful advice during measurements.

\section{References}

1. H.E. Bömmel, K. Dransfeld: Phys. Rev. Letters 1, 234 (1958)

2. W.Eisenmenger, A.H. Dayem: Phys. Rev. Letters 18, 125 (1967)

3. R.J.Gutfeld, A.H. Nethercot,Jr.: Phys. Rev. Letters 12, 641 (1964)

4. O.Weis: Z. Angew. Physik 26, 325 (1969)

5. W.A.Little: Can. J. Phys. 37, 334 (1952)

6. D. Waldmann: Verhandlungen der DPG 8/1974 (Physik Verlag Weinheim 1974) and D.Waldmann: Diplomarbeit, Stuttgart (1973)

7. W. Kappus, O.Weis: J. Appl. Phys. 44, 1947 (1973)
8. H.J.Maris: J. Physique C4, 3 (1972). In contrast to the nonequilibrium properties between electrons and phonons at higher heater powers predicted by N.Perrin, H. Budd: J. Physique C4, 33 (1972) we assume perfect thermal equilibrium, since our experiment concerns only low heater powers

9. Ch.Kittel: Introduction to Solid State Physics, 2nd ed. (John Wiley and Sons, New York 1956) pp. 128

10. G.W.Farnell, E.L.Adler: In Phys. Acoustics, vol. 9, ed. by W.P.Mason, Academic Press, New York (1972) pp. 35

11. W.Eisenmenger: In Tunneling Phenomena in Solids, ed. by E.Burstein, S.Lundquist (Plenum Press, New York 1969) pp. $371-384$

12. H.J.Trumpp, K. Laßmann, W.Eisenmenger: Phys. Letters 41 A, $431(1972)$

13. V.M. Bobetic: Phys. Rev. 136A, 1535 (1964)

14. A.B.Pippard: Phil. Mag. 46, 1104 (1955)

15. J.R. Leibowitz: Phys. Rev. 133 A, 84 (1964)

16. The emissivities $e_{\sigma}^{(10)}$ for a given polarization have been calculated by Weis [4] by averaging over the different directions of the crystal half-space. Because the phonon transmission is limited to a small cone within the critical angle of total reflection, the dependence of $e_{\sigma}^{(10)}$ from the different models is small compared to the influence of the phonon density of states. So we took for all models the same value of $e_{\sigma}^{(10)}$ 